\title{
A REFERENCIALIZAÇÃO NA PESQUISA EM EDUCAÇÃO EM
} CIÊNCIAS

\author{
Bárbara Grace Tobaldini de Lima \\ Graziela Piccoli Richetti \\ José de Pinho Alves-Filho
}

\begin{abstract}
Resumo
Neste trabalho analisamos, a partir da proposta teórica e metodológica da Referencialização, a formação dos egressos de um curso de licenciatura e de um programa de pós-graduação. No primeiro momento abordamos os encaminhamentos e fundamentos deste processo e o modo como ele foi mobilizado no desenvolvimento de duas investigações. O objetivo é apresentar e difundir a referencialização como alternativa para o desenvolvimento das pesquisas na educação em Ciências. Como resultados são apresentadas as dimensões identificadas em cada uma das pesquisas, e em seguida, uma síntese dos principais resultados. Concluímos que a referencialização é um processo dinâmico, complexo, processual e que, ao fornecer respostas, gera também informações para nortear futuras mudanças ou adaptações ao dispositivo educativo analisado.
\end{abstract}

Palavras-chave: formação de professores; avaliação; educação em ciências.

\section{REFERENCIALIZATION IN RESEARCH IN SCIENCE EDUCATION}

\section{Abstract}

In this work, we analyze the egressed students' formation of a degree course and a postgraduate program from the theoretical and methodological proposal of Referentialization. At first, we approached the referrals and theoretical fundaments of this process and the way it was mobilized in the development of two investigations. The objective is to present and spread referentialization as an alternative for the development of research in science education. As results, the dimensions identified in each of the surveys are presented, and then a summary of the main results. We conclude that referentialization is a dynamic, complex process that can provide some answers, in addition to generating information to guide future changes in the course.

Keywords: teacher education; evaluation; science education.

\section{LA REFERENCIALIZACIÓN EN LA INVESTIGACIÓN EN EDUCACIÓN EN CIENCIAS}

\section{Resumen}

En este trabajo se analiza, desde la propuesta teórica y metodológica de la Referencialización, la formación de los egresados de un curso de profesorado y de un programa de posgrado. En un primer momento abordamos las direcciones y fundamentos de este proceso y la forma como fue movilizado en el desarrollo las investigaciones. El objetivo con este trabajo es presentar y difundir la referencialización como alternativa para el crecimiento de las investigaciones en la Educación en Ciencias. Como resultados se presentan las dimensiones identificadas en cada una de las encuestas y una síntesis de los principales resultados. Concluimos que la referencialización es un proceso dinámico, complejo, procesal y que al dar respuestas también genera informaciones para orientar futuros cambios o adaptaciones al dispositivo educativo analizado.

Palabras clave: formación de profesores; evaluación; educación en ciencias. 
DOI: $10.12957 /$ teias. $\%$ Y.51288

\section{INTRODUÇÃO}

A qualidade na formação de professores para o ensino na educação básica e no ensino superior é recorrente nos debates, em palestras e conferências, anais de eventos, publicações em periódicos e discussões políticas (DINIZ-PEREIRA, 2000; PERRENOUD, 2002; CACHAPUZ, 2005; GATTI, 2010; CUNHA, 2013). Nesses trabalhos há argumentos a respeito dos modelos e práticas formativas, objetivos e finalidades da educação, das práticas formativas para atuação profissional, como também nos encaminhamentos e propostas das políticas públicas educacionais. No entanto, em que medida os argumentos a respeito da formação de professores são implementados no cenário da educação básica e do ensino superior?

A expansão dos programas de pós-graduação, o apoio ao desenvolvimento científico, a internacionalização, a promoção de eventos científicos nacionais e internacionais, os grupos de pesquisa, entre outras redes colaborativas, vêm contribuído para fortalecer a produção do conhecimento científico sobre as particularidades do sistema público de educação. No entanto, quais resultados produzidos por essas pesquisas podem ser incluídos no currículo das escolas de educação básica, por exemplo, ou nos cursos de formação de professores e na pós-graduação? Diante das críticas, limitações e ordenamentos superiores aos espaços educacionais e aos seus atores, como os cursos de licenciatura e da pós-graduação podem se manifestar frente a esse contexto?

Para nós, a resposta a esses questionamentos envolve um conjunto de aspectos teóricos, reflexivos, reais, contraditórios, pedagógicos e tantos outros que culminam nas diferentes possibilidades formativas dos cursos de graduação e pós-graduação. Da mesma forma nas compreensões do professor e no desenvolvimento das pesquisas. Essa diversidade, mais as particularidades de cada projeto institucional, precisam ser consideradas quando se propõe analisá-los. Para isso, encontramos na literatura uma proposta teórica e metodológica que considera o processo de avaliação de modo amplo, global e inclusivo das diferenças e características peculiares do "objeto/fenômeno" a ser investigado - a Referencialização, proposta por Gerald Figari (1996). Com apoio da Referencialização, é nosso objetivo apresentar e explorar as suas potencialidades a partir da análise de dois cursos, um de graduação e outro da pósgraduação com formação na educação em Ciências.

\section{A AVALIAÇÃo NA PERSPECTIVA DA REFERENCIALIZAÇÃo}

A Referencialização, proposta no livro "Avaliar: que referencial?” de Gerald Figari em 1996, é muito explorada no contexto português pelas Universidades de Coimbra, Universidade de Lisboa, Universidade de Aveiro e a Universidade do Minho, a exemplo das teses de doutorado produzida por Lopes (2011), Magueta (2012) e Guerreiro (2015) e dos artigos publicados em atas de eventos como Alves e Correia (2009) e Ramalho (2014).

No Brasil, identificamos no Catálogo de Teses e dissertações da Coordenação de Aperfeiçoamento de Pessoal de Nível Superior (CAPES) 13 trabalhos que fizeram o uso da Referencialização. No entanto, apenas quatro delas estão relacionadas de alguma forma com a educação em Ciências: duas dissertações (VICENTE, 2007; PAIVA, 2016) e duas teses (BRAGA, 2001; RICHETTI, 2014). Recentemente, a tese defendida por Lima (2019), também utilizou a referencialização.

Figari (1996) assinala que esse método se destina à avaliação das estruturas educativas, caracterizadas como espaços ou sistemas para o desenvolvimento de competências e 
qualificações de natureza educacional ou formativa. As estruturas educativas podem ser tratadas como: macroestrutura, quando o sistema é o objeto a ser estudado; microestruturas, ao se referir a um grupo de formação, e mesoestrutura, representada pelos espaços de ensino e formação, como as escolas e universidades. Contudo, o autor adverte que o termo "estrutura" pertence mais ao campo social e político do que ao pedagógico, e que, por estar mais interessado nas mesoestruturas, o termo "Dispositivo" torna-se mais adequado, porém "[...] não é uma simples categoria descritiva dos sistemas de formação, mas que é, em si mesmo 'educativo' quando desempenha um papel formador." (FIGARI, 1996, p. 30).

O Dispositivo Educativo (DE) é o resultado de um processo de construção coletiva e foi um projeto de aprendizagem a respeito dos conteúdos, das competências e tarefas. Essa aprendizagem pode ser fruto de um projeto de longo prazo e localizado em estabelecimentos escolares, organismos internos de formação, ocasional e circunstancial, quando realizado em ciclos de formação ou encontros para formação dos formadores.

Ao considerar as diferentes características e a complexidades de um DE, Figari (1996) propõe alguns questionamentos em que as respostas conduzem às três dimensões intrínsecas de um DE e relacionam-se aos três momentos formativos da avaliação.

1) Como tratar as determinações que induzem as escolhas educativas, que justificam a prática deste ou daquele dispositivo? 2) Como explicar, acompanhar e orientar a elaboração dos projetos, dos programas, dos currículos? 3) Como observar, por fim, e interpretar as formas evidentes, e mesmo dadas a ver, desta ação assim como os seus efeitos, isto é, a sua produção? (FIGARI, 1996, p. 32).

Para o primeiro questionamento, são apresentados os procedimentos que permitirão oferecer as informações sobre o meio, as características dos envolvidos no sistema avaliado e as informações diagnósticas e gerais sobre a situação a ser estudada. Esse conjunto nomeia-se: Dimensão Induzida. Ao segundo questionamento, almeja-se uma melhor compreensão do DE, analisando os processos que definem a ação educativa, a dimensão nomeada de "Construído". O terceiro questionamento enfatiza a produção decorrente das outras dimensões e apresenta um conjunto de informações, as quais caracterizam a última dimensão, ou seja, aquilo que foi "Produzido". Esse sistema e o modo de olhar para o DE inquietam Figari, no sentido de: "Como será possível aceder a um sistema de referências e, em seguida, a um modelo de compreensão dos dispositivos educativos que permita afirmar que estes últimos obedecem a uma lógica própria, que produzem efeitos que lhe são próprios?” (FIGARI, 1996, p. 60).

As três dimensões citadas anteriormente são utilizadas para modelar o DE, que devem ser compreendidas de modo interativo e integrativo, como representado por Figari (1996). Agregamse a essas dimensões, segundo Figari e Tourmen (2006), três tipos de informações que podem atuar e interferir entre si: dados de entrada, relacionados às origens, os elementos contextuais e os propósitos; informações sobre os processos, negociações e as construções e, finalmente, dados sobre produtos, resultados e/ou efeitos produzidos pelo DE.

Em resumo, a dimensão do Induzido (I) contempla os dados da entrada; no Construído (C), as informações que demonstram os processos; e no Produzido (P), os resultados identificados. Figari e Tourmen (2006) consideram que é possível avaliar um DE a partir das relações entre as três dimensões. A avaliação entre o I e o C permite estudar se os objetivos do $\mathrm{DE}$ são adequados às estratégias construídas; entre I e $\mathrm{P}$, podemos avaliar a rentabilidade do programa, ou seja, se o DE é coerente com os seus objetivos e, por último, a eficiência entre C e 
$\mathrm{P}$, para avaliar se as estratégias utilizadas são válidas para o resultado que se espera. Portanto, a partir deste modelo, podemos avaliar a relevância (I-C), a rentabilidade (I-P) e a eficiência (C-P) de um DE.

A partir do "Induzido", o avaliador utilizará fontes que possibilitem a descrição dos dados do contexto investigado e as suas origens. É o ponto de entrada para a elaboração do quadro de referências que, por sua vez, determinará as origens do DE. A finalidade da avaliação é diagnosticar e interpretar as informações que se colocam ao contexto que será avaliado. No "Construído", busca-se compreender as características, concepções e finalidades, que estão relacionadas e são influenciadas pelo polo anterior. Na perspectiva da avaliação, assume-se a função de regulação, pois visa a acompanhar o processo de elaboração.

No "Produzido", estarão as informações obtidas a partir de um conjunto de elementos metodológicos, que serão analisadas e interpretadas pelo pesquisador a partir das produções realizadas nas dimensões anteriores. Considerando as três dimensões e os seus principais elementos, Figari (1996) considera que o esquema ICP permitirá ao pesquisador elaborar um modelo do DE, ou seja, um sistema abstrato, uma descrição daquilo que foi idealizado e que caracteriza a situação que é estudada e investigada. Em outro momento, esse modelo permitirá entender e interpretar a realidade, mediante as informações da dimensão Produzido.

Para Figari (1996, p. 63), “[...] o esquema ICP é apenas o resultado, absolutamente provisório, de uma tentativa de se dispor de um princípio explicativo do funcionamento dos dispositivos educativos". Com base em suas articulações e no cenário criado, o esquema pode ser aplicado para três ações distintas: a) descrever o funcionamento de um DE em torno de um sistema de dimensões; b) relacionar a avaliação de um DE com um modelo; c) construir um referencial específico para cada DE.

O esquema ICP permite compreender o DE e produzir afirmações que são lógicas e inerentes a ele, bem como analisar aquilo que é próprio da natureza investigada. Além disso, colabora para responder ao questionamento central da referencialização: o que se pretende avaliar? Esse questionamento apresenta dois encaminhamentos: o primeiro é de ordem teórica, pois só podemos avaliar aquilo que já deu lugar para a descrição de suas finalidades, seus princípios, objetivos e suas especificidades, um “[...] objeto de que qualquer forma já conceptualizado." (FIGARI, 1996, p. 36). Entretanto, se levarmos em consideração a complexidade do que se avalia, como chegar a essas informações? Isso conduz ao segundo encaminhamento, de ordem metodológica, pela necessidade de construir um quadro de referências para auxiliar no reconhecimento das informações necessárias para determinar o que se pretende avaliar (FIGARI, 1996).

Para compreender o que é e como se constrói um quadro de referências, consideramos como oportuno apresentar três conceitos centrais e relacionados diretamente a ele: referente; referido e referencial. Ao mencionarmos os dados de entrada, as informações que modelam o abstrato, os objetivos desejados, estamos falando nos "referentes" da pesquisa, materiais ou documentos que nos auxiliarão a criar um modelo do ideal (HADJI, 1994). O "referido", por sua vez, está na esfera da realidade observada, uma representação dos fatos, daquilo que diz respeito ao referente, mas no âmbito do real, do concreto e não mais das expectativas ou do idealizado, como é no referente (HADJI, 1994; FIGARI, 1996). E será da articulação entre o referente (as normas, o modelo, o objetivo esperado) e o referido (o que é constatado, objeto de investigação) que se realizará a avaliação do DE.

O termo referencial, por sua vez, pode ser utilizado como sinônimo de quadro ou sistema de referência e apresenta três características essenciais. A primeira é derivada do plano operatório, 
quando se constrói um instrumento, que permita, por exemplo, observar um currículo. No plano metodológico, segunda característica, o referencial justifica um conjunto de procedimentos e as finalidades das estratégias utilizadas. E a terceira trata do plano científico, o referencial ou o quadro de referências que oferecerá ao pesquisador um "[...] corpo de hipóteses que preveja a explicação dos resultados." (FIGARI, 1996, p. 58). Nesse entendimento, ele assume um caráter estático, o qual é corrigido para referencialização de modo a demonstrar que o sistema de referência é construído durante a investigação.

A Referencialização consiste na elaboração de um quadro de referências, que irá descrever e modelar inicialmente o $\mathrm{DE}$ e o objeto de estudo. $\mathrm{Na}$ sequência, fornecerá informações orientadoras para que ele seja avaliado. Destacamos, assim, que uma das potencialidades deste método de investigação é que, ao avaliar um DE a partir da Referencialização, criam-se objetivos, critérios e indicadores próprios da situação investigada, do caso estudado, preservando suas características e peculiaridades. A Referencialização é, portanto, abstrata e flexível, por modelizar e assumir as caraterísticas do DE e do objeto avaliado.

A partir do quadro de referências, identificaremos um campo científico para legitimar, investigar e explicar o objeto de estudo, as escolhas teóricas e metodológicas, esclarecer o campo de investigação, justificar os critérios e outros elementos necessários para avaliar o DE. Assim, a referencialização é utilizada para avaliar um modelo de DE a partir de diferentes olhares e perspectivas, e também colabora no desenvolvimento, na construção, problematização e explicação do objeto avaliado. "[...] A Referencialização consiste em assinalar um contexto e em construir, fundamentando-o com os dados, um corpo de referências relativas a um objeto (ou a uma situação), em relação ao qual poderão ser estabelecidos diagnósticos, projectos de formação e avaliações." (FIGARI, 1996, p. 52).

Em linhas gerais, o método respeita a identidade e os interesses dos avaliados, realiza uma análise criteriosa e metódica dos materiais que são fonte de informação e os resultados oriundos podem fornecer elementos para apoiar futuros trabalhos de intervenção. Mediante os conceitos de DE, referente, referido e referencial e da dimensão do Induzido, Construído e Produzido (ICP), admitimos a Referencialização como um método capaz de orientar a leitura da realidade para avaliar a formação dos egressos de um curso de licenciatura e da pós-graduação, seguindo os objetivos e princípios relativos à instituição formadora.

\section{Elementos para a construção da referencialização}

Para a construção do referencial de avaliação - a Referencialização -, Figari (1996) sinaliza, basicamente, a necessidade de quatro fases: a) a origem da avaliação; b) o projeto de avaliação; c) o plano de estudo; d) a construção do referencial e da Referencialização.

A primeira fase, a "origem da avaliação", é, segundo Figari (1996), o pedido de investigação apresentado a um avaliador. $\mathrm{Na}$ segunda fase, o "projeto de avaliação", realiza-se um metaprojeto, em que são definidas a organização, a especificidade do objeto de estudo e do DE, as questões que envolvem a ordem metodológica do trabalho de pesquisa, as estratégias e os instrumentos de pesquisa e os métodos de análise que serão utilizados para interpretar as informações produzidas em cada um dos momentos das pesquisas.

A terceira fase consiste na organização de um "plano de estudo", em que seu objetivo é “[...] transformar as questões do pedido em questões de avaliação, ou seja, passar do plano estratégico para o plano científico [...]" (FIGARI, 1996, p. 139). É uma ação de organização de um plano de descrição que permita ao avaliador ter, não apenas uma descrição daquilo que é 
visível, mas também uma lista de questões que se colocam à avaliação. Para chegar a este plano de estudo, são sugeridas quatro etapas: a) dimensões do problema; b) ajustamento das questões; c) linhas da investigação e construção de um corpo de materiais; e d) modos de tratamento e análise.

A quarta e última fase da construção do referencial de avaliação consiste na construção do referencial e da Referencialização, que busca responder como os dados obtidos a partir das etapas anteriores podem dar lugar à formação de categorias de um referencial. O referencial é um retrato pontual e selecionado da Referencialização, um documento ou um quadro de referências que acompanha a avaliação do DE. É um documento que organiza, sistematiza o que já foi construído e aponta para elementos que ainda precisam ser elaborados ou desenvolvidos envolvendo os referentes, as dimensões, os instrumentos e os métodos que produzem as informações do que foi analisado, e indica os caminhos para realizar a avaliação.

\section{ANÁLISE DE DISPOSITIVOS EDUCATIVOS À LUZ DA REFERENCIALIZAÇÃO}

Na pesquisa desenvolvida no âmbito dos cursos de licenciatura (LIMA, 2019), a origem da avaliação encontra-se no problema de pesquisa que foi elaborado da seguinte forma: Como os objetivos e os princípios da formação docente preconizados pela Universidade Federal da Fronteira Sul (UFFS) estão presentes na formação dos egressos licenciados em Ciências Biológicas? Deste questionamento, outros são decorrentes e permitem encaminhar teórica e metodologicamente a pesquisa. Nesta fase, os questionamentos, os objetivos e outros encaminhamentos gerais da pesquisa colaboram para a delimitação do objeto de estudo e para a elaboração do quadro de referências.

$\mathrm{Na}$ segunda fase, Lima (2019) buscou compreender a organização dos cursos de licenciatura no país, e para isso, recorreu a trabalhos que, ao relatarem as raízes históricas desses cursos, auxiliassem a compreender as preocupações e finalidades envolvidas na formação de professores. A partir deste movimento, foram realizados os primeiros questionamentos para orientar a análise dos referentes externos e internos, e por meio de uma análise exploratória identificar as dimensões que envolvem o DE.

No plano de estudo, terceira fase da Referencialização, Lima (2019) realizou alguns questionamentos e com eles identificou as características, finalidades, objetivos, e as estratégias do DE, e em seguida a construção de um plano de investigação sobre as informações identificadas. Nesse momento, compreendeu que as informações iniciais sobre ações desenvolvidas por um curso de graduação poderiam estar nos documentos institucionais e no discurso dos professores. Essas possibilidades encaminham para outro momento, quando é identificada e apresentada o modo como as informações serão produzidas e analisadas. Para isso, a autora optou pela Análise Documental, e pelo uso da Análise Textual Discursiva.

Finalmente, na última fase, a construção do referencial de avaliação, Lima (2019) identificou as dimensões do seu referencial e que nortearam a investigação, sendo elas: a Dimensão Curricular, Avaliativa, Ciência e Sociedade, Profissional e Valores. Essas dimensões foram, portanto, identificadas a partir do processo de Referencialização, representado a partir do esquema presente na Figura 1. 
Figura 1: Esquema representativo da Referencialização

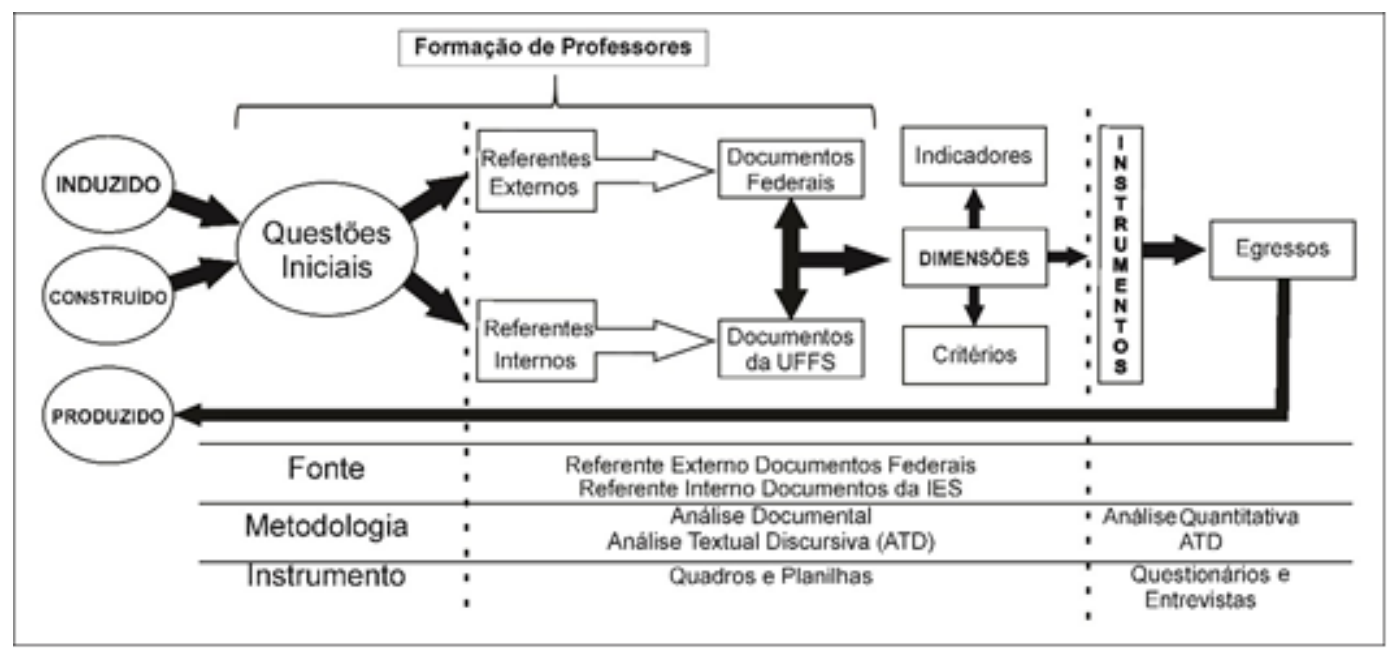

Fonte: Lima (2019, p. 44)

Com as informações presentes no questionário e depois investigadas na entrevista, destacamos os principais resultados que foram encontrados, entre eles: que as técnicas expositivas e tradicionais ainda são corriqueiras, hegemônicas, pode-se dizer, nas salas de aula, sendo utilizadas tanto pelos formadores quanto pelos acadêmicos. No tangente à prática da leitura, vê-se que ela se caracteriza pelo uso de textos técnicos, com temas pontuais, e relacionados, principalmente, às atividades avaliativas. $\mathrm{E}$ como muitos acadêmicos trabalham durante o dia e não há estímulo para o ato de ler, eles se distanciam de uma prática de leitura mais significativa e formativa.

Além disso, Lima (2019) não identificou, na análise das entrevistas, atividades e estratégias de ensino relacionadas à promoção de conhecimento sobre as Tecnologias de Informação e Comunicação (TIC) e com a abordagem de conteúdos atitudinais. No que se refere às práticas interdisciplinares, a autora também constatou a necessidade de que sejam realizadas formações aos docentes e acadêmicos quanto à promoção de diálogo entre os formadores, à escolha das temáticas a serem investigadas e à discussão sobre o que é interdisciplinaridade.

As informações obtidas a partir da investigação da dimensão "Avaliativa" demonstram que uma abordagem teórica sobre a avaliação da aprendizagem foi promovida. No entanto, os entrevistados deixaram evidente que o principal instrumento utilizado foi a "prova" e, na sequência, citaram as limitações deste instrumento. E, na dimensão Profissional, as informações exploradas por Lima (2019) indicam que em relação ao contexto educacional das diferentes modalidades e níveis de ensino, a formação dos participantes esteve centralizada nas escolas de educação básica localizadas no meio urbano. Das possibilidades, poucas foram as discussões e as inserções nas escolas do Campo e na Educação de Jovens e Adultos. A Educação Especial foi abordada principalmente em questões teóricas, pelo Componente Curricular (CCR) destinado a esta abordagem. Porém, a partir dos referentes, a autora ressalta que a formação dos acadêmicos deveria contemplar o trabalho com as modalidades já citadas, além das escolas quilombolas e indígenas e do ensino noturno. Esses são apenas alguns dos resultados encontrados por Lima (2019), mas que no conjunto indicam a necessidade de que os referentes sejam conhecidos, discutidos e explorados junto aos acadêmicos, formadores e gestores da instituição. 
No trabalho de Richetti (2014), a Referencialização foi utilizada para analisar quais aspectos têm favorecido o êxito dos cursos de mestrado e doutorado do Programa de PósGraduação em Educação Científica e Tecnológica da Universidade Federal de Santa Catarina (PPGECT/UFSC), bem como os elementos que questionam seus limites. A origem da avaliação foi apresentada no problema de pesquisa: A formação de mestres e doutores está harmônica com os objetivos propostos pelo PPGECT/UFSC e faz deles indivíduos comprometidos em sua atuação pedagógica/profissional e multiplicadores dos saberes adquiridos?

$\mathrm{Na}$ primeira etapa realizou-se um estudo exploratório sobre o surgimento da pósgraduação (PG) brasileira, desde a década de 1930 até a constituição da "Área 46", no ano 2000. Inicialmente, o sistema da PG era predominantemente disciplinar, mas com o passar dos anos abriu-se para a interdisciplinaridade e multidisciplinaridade, direcionando-se para a importância da inter-relação entre conteúdo, ensino e didática. Também foram realizadas entrevistas com os professores que projetaram o PPGECT/UFSC e seus relatos sobre o contexto, as necessidades e as expectativas que deram origem ao Programa revelaram o caráter pioneiro do curso e a forte componente epistemológica presente em sua estrutura curricular. Esses dados referem-se às origens e constituem a dimensão do Induzido. Já a dimensão do Construído congrega os aspectos históricos relacionados ao desenvolvimento da PG brasileira, influenciado por leis e decretos específicos que estruturaram, normatizaram e orientaram o funcionamento dos cursos. Finalmente, a dimensão do Produzido foi organizada com os dados oriundos da análise das dissertações e teses do PPGECT/UFSC e pelos dados empíricos obtidos com os egressos por meio de questionários e entrevistas, que apresentaram o impacto dos cursos de mestrado e doutorado na atuação profissional dos egressos.

A primeira parte do referencial contém uma lista de critérios de avaliação extraídos dos referentes externos constituídos pela Lei de Diretrizes e Bases da Educação, os Planos Nacionais de Pós-Graduação e as Avaliações Trienais da CAPES. Por sua vez, os referentes internos congregaram os documentos publicados pela UFSC, como a resolução sobre a PG, o regimento e o caderno de indicadores do PPGECT/UFSC (RICHETTTI, 2014).

A análise dos referentes internos e externos conduziu à construção da segunda parte do referencial, resultando nas dimensões da avaliação do PPGECT/UFSC: Curricular, relacionada aos objetivos propostos para a formação de mestres e doutores; Epistemológica, alusiva à apreensão de novos conhecimentos, mudanças ou refinamento dos já existentes; Educação Científica e Tecnológica, no tocante às correlações entre educação e ciência, educação e tecnologia e os desdobramentos no contexto social e Atuação Pedagógica/Profissional, relativa às atividades formativas e sua importância para a atuação dos egressos. O esquema geral do percurso da referencialização encontra-se na Figura 2: 
Figura 2 - Esquema representativo do percurso da Referencialização - PPGECT/UFSC.

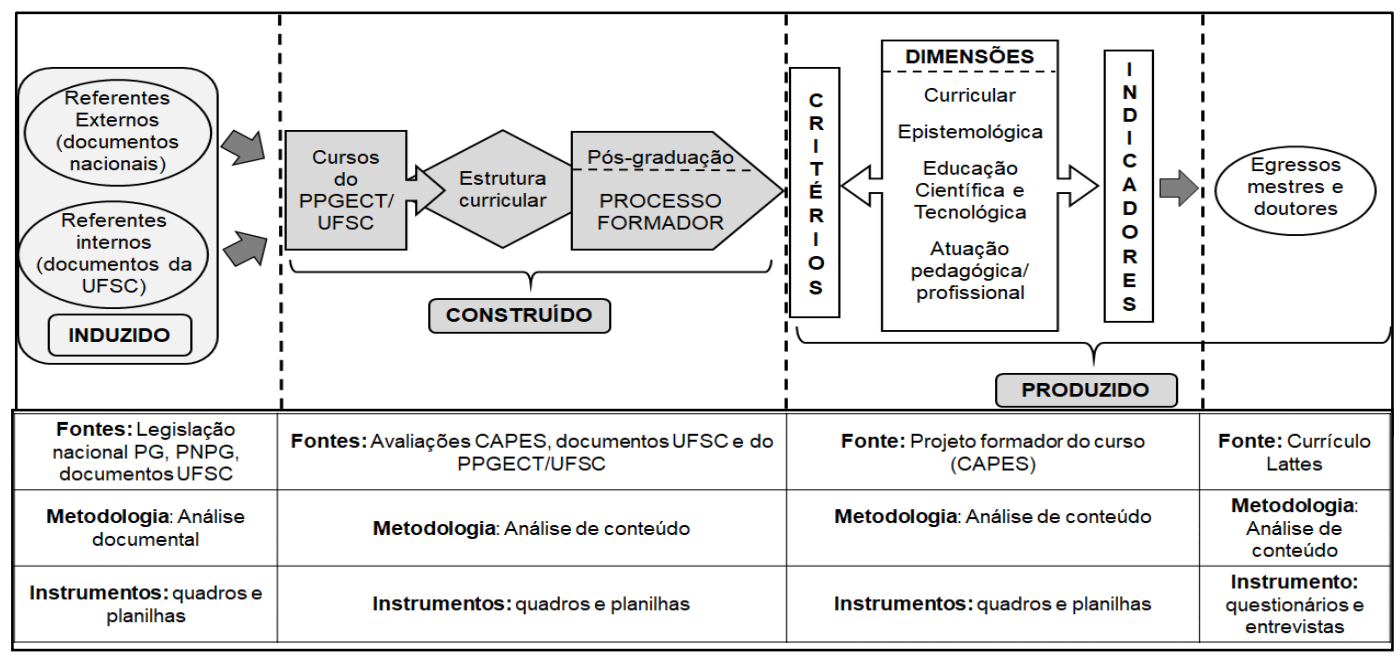

Fonte: adaptado de Richetti (2014, p. 148)

Os dados dos questionários respondidos pelos egressos mestres e doutores apontaram que ambos os cursos têm gerado efeitos positivos significativos em sua atuação profissional, entre os quais, a elaboração de concepções fundamentadas sobre educação, ciência, ensino e aprendizagem. Merecem destaque os aspectos relacionados à autonomia e à segurança para o desenvolvimento de atividades acadêmicas, apontados pelos mestres e doutores como muito relevante durante a formação. Entre as limitações apontadas, a principal diz respeito à distância existente entre as pesquisas desenvolvidas nas dissertações e teses e a realidade da atuação profissional nos contextos educacionais.

As motivações, resultados, contribuições e impactos, bem como as sugestões dos egressos para o programa, mostraram que os cursos trouxeram benefícios concretos à própria atuação pedagógica. As atividades formativas foram percebidas como meios efetivos para reelaboração das concepções sobre a construção do conhecimento científico e, consequentemente, para pensar a respeito da sua identidade profissional. Para os egressos mestres e doutores, o estudo da epistemologia da ciência foi fundamental para sua formação e atuação pedagógica/profissional por proporcionar um melhor entendimento sobre a construção do conhecimento científico.

\section{CONSIDERAÇÕES FINAIS}

Ao analisar a formação dos egressos, a partir da Referencialização, e em diferentes níveis e modalidades de ensino, consideramos que essa proposta teórica e metodológica com seus critérios e indicadores permite uma avaliação mais qualificada a respeito da formação, tanto dos licenciados em Ciências Biológicas como dos mestres e doutores em Educação Científica e Tecnológica. No caso da avaliação do curso de graduação, emerge a necessidade de promover uma formação permanente aos formadores, de modo que eles se compreendam como formadores de um curso de licenciatura, não esquecendo que suas práticas refletem uma concepção a respeito da ação pedagógica. Olhar e promover uma formação permanente junto aos docentes formadores é compreender a complexidade que envolve a docência, as mudanças na sociedade, com os desafios do trabalho pedagógico. É preciso compreender que as orientações e 
as recomendações para o trabalho junto à educação básica também perpassam a educação superior.

Partindo do princípio de que os documentos institucionais são elaborados em atendimento aos documentos nacionais é que afirmamos que as concepções que os cursos em tela estabeleceram como princípios e objetivos para a formação de seus egressos refletem o que está normatizado nos documentos nacionais. São discussões e orientações que não se restringem apenas a esses espaços, elas também estão presentes há décadas na pesquisa educacional, com sugestões significativas à formação de professores. Portanto, os princípios e os objetivos precisam ser reconhecidos, trabalhados e mobilizados por todos aqueles que fazem parte da formação de novos profissionais da educação. Ademais, os efeitos das atividades formativas serão refletidos na atuação pedagógica/profissional, multiplicando-se em diferentes contextos e contribuindo para a melhoria do ensino de Ciências.

\section{REFERÊNCIAS}

ALVES, Maria Palmira Carlos; CORREIA, Serafim Manuel Teixeira. Projecto de Avaliação em Rede (PAR): Construção e regulação do sentido colectivo de escola. In: Congresso Internacional Galego-Português de Psicopedagogia, 10, 2009, Braga. Anais [...] Braga. Universidade do Minho, 2009.

BRAGA, Hilda Maria Cordeiro Barroso. Avaliação da Reformulação Curricular do Curso de Administração: a avaliação de dispositivos educacionais a partir do método de referencialização de Figari. 299 f. Tese (Doutorado em Educação), Pontifícia Universidade Católica de São Paulo, São Paulo, 2001.

CACHAPUZ, Antonio. et al. A necessária renovação do ensino de Ciências. São Paulo: Cortez, 2005.

CUNHA, Maria Isabel. O tema da formação de professores: trajetórias e tendências do campo na pesquisa e na ação. Revista Educação e Pesquisa, São Paulo, v. 39, n. 3, p. 1-17, 2013.

DINIZ-PEREIRA, Júlio Emilio. A formação acadêmico-profissional: compartilhando responsabilidades entre universidades e escolas. In: Encontro Nacional de Didática e Prática de Ensino, 14, 2008, Porto Alegre. Anais [...] Porto Alegre: PUCRS, 2008.

FIGARI, Gerald. Avaliar: que referencial? Trad. Júlia Lopes Ferreira e José Manuel Cláudio. Porto: Porto, 1996.

FIGARI, Gerald., TOURMEN, Claire. La référentialisation: une façon de modéliser l'évaluation de programme, entre théorie et pratique Vers une comparaison des approches au Québec et en France. Mesure et évaluation en éducation, Canadá, v. 29, n. 3, p. 5-25, 2006.

GUERREIRO, Helder Lopes Para a compreensão da sustentabilidade da autoavaliação das escolas: um estudo de caso múltiplo em escolas públicas portuguesas. 284 f. Tese (Doutorado em Educação), Lisboa, 2015.

HADJI, Charles. A Avaliação, regras do Jogo: das intenções aos instrumentos. Trad. Júlia Lopes Ferreira e José Manuel Cláudio. Porto: Porto, 1994.

LIMA, Bárbara Grace Tobaldini. Avaliação da formação inicial por egressos licenciados da UFFS: o curso de Ciências Biológicas como estudo de caso, 290 f. Tese (Doutorado em Educação Científica e Tecnológica), Universidade Federal de Santa Catarina, Florianópolis, 2019.

LOPES, Teresa Cristina Teles. Contribuição da avaliação formativa para o desenvolvimento cognitivo em alunos de Física. 49 f. Projeto de Tese. Universidade de Coimbra, Coimbra, 2011. 
MAGUETA, Lucia Grava. Um estudo de avaliação do currículo da área de expressão e educação plástica no $1^{\circ}$ ciclo do ensino básico com base na metodologia da referencialização. $310 \mathrm{f}$. Tese (Doutorado em Educação), Instituto de Educação. Lisboa, 2012.

PAIVA, Camila. Avaliação da promoção da alfabetização científica e tecnológica em vivências de ilha interdisciplinar de racionalidade. 269 f. Dissertação (Mestrado em Educação Científica e Tecnológica), Universidade Federal de Santa Catarina, Florianópolis, 2016.

PERRENOUD, Phillippe. As competências para ensinar no século XXI: A formação dos professores e o desafio da avaliação. Porto Alegre: Artmed, 2002.

RAMALHO, Henrique. Contributos Teóricos e Metodológicos Para a Referencialização da Avaliação do Desempenho do Docente. Revista Eletrônica de Educaşão e Psicologia, Portugal, n. 1, p. 12-21, 2014.

RICHETTI, Graziela Piccoli. Um instrumento para avaliar a formação de egressos da pós-graduação: o programa de pós-graduação em Educação Científica e Tecnológica da Universidade Federal de Santa Catarina como estudo de caso. 412 f. Tese (Doutorado em Educação Científica e Tecnológica), Universidade Federal de Santa Catarina, Florianópolis, 2014.

VICENTE, Maria Heloisa Saraiva. Táticas de professores: uma reflexão sobre o cenário avaliativo no regime de progressão continuada. 126 f. Dissertação (Mestrado em Educação), Universidade Metodista de São Paulo, São Paulo, 2007.

GATTI, Bernadete. A. Formação de professores no Brasil: características e problemas. Educação e Sociedade, v. 31, n. 113, p. 1355-1379, 2010.

\section{Informações dos autores}

Bárbara Grace Tobaldini de Lima

Universidade Federal da Fronteira Sul

E-mail: tobaldinibg@gmail.com

ORCID: https://orcid.org/0000-0002-6502-7306

Link Lattes: http://lattes.cnpq.br/ 6819394499327864

Graziela Piccoli Richetti

Universidade Federal de Santa Catarina

E-mail: graziela.richetti@ufsc.br

ORCID: https://orcid.org/0000-0001-9868-7768

Link Lattes: http://lattes.cnpq.br/9128328286908246

José de Pinho Alves-Filho

Universidade Federal de Santa Catarina

E-mail: jopinhofilho@gmail.com

ORCID: https://orcid.org/0000-0002-6407-4418

Link Lattes: http://lattes.cnpq.br/5918827024479712 